\title{
Representações sociais da violência sexual na produção científica nacional'
}

\author{
Social representations of sexual violence in the national scientific literature
}

\author{
Arielle Sagrillo Scarpati ${ }^{[a]}$, Edinete Maria Rosa ${ }^{[b]}$, Valeschka Martins Guerra ${ }^{[c]}$
}

\footnotetext{
${ }^{[a]}$ Mestre em Psicologia pela Universidade Federal do Espírito Santo, Vitória, ES - Brasil, e-mail: arielle.psicologia@gmail.com

${ }^{[b]}$ Doutora em Psicologia Social, professora da Universidade Federal do Espírito Santo, Vitória, ES - Brasil, e-mail:

edineter@gmail.com

${ }^{[c]}$ Doutora em Psicologia Social, professora da Universidade Federal do Espírito Santo, Vitória, ES - Brasil, e-mail: valeschkamartins@googlemail.com

${ }^{[1]}$ Nota dos autores: 0 presente estudo contou com o apoio de uma Bolsa de Demanda Social concedida ao primeiro autor pela CAPES, instituição que os autores agradecem.
}

Recebido: 14/04/2012 Received: 04/14/2012

Aprovado: $21 / 11 / 2012$ Approved: 11/21/2012

\section{Resumo}

Esse trabalho teve como objetivo identificar as representações sociais da violência sexual contra a mulher adulta na produção científica nacional sobre o tema. Para tanto, foi realizada uma pesquisa bibliográfica, que revisou os artigos indexados na Scientific Electronic Library - Scielo dos últimos cinco anos, que continham pelo menos uma das seguintes palavras-chave: violência sexual, abuso sexual, estupro e violência contra a mulher. Primeiramente, foram mapeadas as datas de publicação, áreas de conhecimento e vinculação institucional do primeiro autor dos artigos. Em seguida, foi realizada a análise dos objetivos gerais dos artigos a partir da Análise de Conteúdo Temática. Os resultados indicam que as RS da violência sexual contra a mulher se organizam em torno do elemento Atendimento, sendo enfatizadas as publicações da área da saúde, centralizadas no Estado de São Paulo. Discute-se a ausência de publicações da Psicologia sobre o tema.

Palavras-chave: Representações Sociais. Violência sexual. Produção acadêmica. Análise de Conteúdo.

\section{Abstract}

This article aimed to identify social representations of sexual violence against adult women in the Brazilian scientific production. A bibliographic research was conducted, reviewing all the articles indexed at the Scientific Electronic Library - Scielo in the last five years, containing at least one of these keywords: sexual violence, sexual abuse, rape and violence against women. Initially, the dates of publication, areas of knowledge and institutional affiliation of the first author were mapped. The second step was to conduct a content analysis of the general objectives of the articles. The results indicated that social representations of sexual violence against women are organized around the element 'Attendance', with emphasis on health-related publications, mainly from the state of São Paulo. The lack of research studies published in Psychology about the topic is discussed.

Keywords: Social Representation. Sexual Violence. Academic production. Content Analysis. 


\section{Introduç̃̃o}

Fenômeno extremamente complexo, a violência possui raízes em quaisquer tipos de relações de poder, sejam com base em questões como a sexualidade, o gênero, a autoidentidade ou nas instituições sociais (Giffin, 1994; Narvaz \& Koller, 2006).

De acordo com Chauí (1985), a violência deve ser compreendida não apenas como violação ou transgressão de regras e leis. Esta deve ser analisada, principalmente, sob dois outros aspectos: em primeiro lugar, enquanto transformação de uma relação de diferença - que não é algo, a priori, ruim - numa relação de desigualdade, de dominação e de exploração; e, em segundo lugar, como qualquer ação que transforme um ser humano em "coisa".

Segundo Araújo (2002, p. 4), a violência é, então, "uma relação de forças caracterizada num pólo pela dominação e no outro pela coisificação", em que o ponto mais importante é a violação do direito de liberdade da vítima. Ou seja, a violação do direito que todo ser humano tem de ser constituinte de sua própria vida, de sua própria história.

Lima, Buchele e Climaco (2008, p. 70) comenta que "[...] diferentes formas de violência, assim como as suas representações, não podem ser encaradas como fenômenos a-históricos e destituídos de subjetividade [...]", uma vez que, ao descartar estes aspectos, tornar-se-ia praticamente impossível a compreensão de toda sua complexidade.

A Organização Mundial de Saúde (2005), por sua vez, define violência como o uso deliberado de força física ou o uso do poder contra outro indivíduo (ou grupo) e que venha a causar algum tipo de lesão ou dano - seja ele físico ou psicológico.

Pensando-se em números, como forma de se exemplificar a relevância deste tema, estima-se que, mundialmente, um em cada cinco dias de afastamento do trabalho solicitado por mulheres decorre de algum tipo de violência sofrida (Narvaz \& Koller, 2006).

Narvaz e Koller (2006, p. 9) ressaltam ainda que:

Na América Latina, a violência doméstica incide entre $25 \%$ e $50 \%$ das mulheres. No Brasil, $23 \%$ das mulheres brasileiras estão sujeitas à violência doméstica; a cada 4 minutos, uma mulher é agredida em seu próprio lar por uma pessoa com quem mantém relação de afeto; $70 \%$ dos crimes contra a mulher acontecem dentro de casa e o agressor é o próprio marido ou companheiro; mais de $40 \%$ das violências resultam em lesões corporais graves decorrentes de socos, tapas, chutes, amarramentos, queimaduras, espancamentos e estrangulamentos.

Tal tipo de estatística requer uma análise cuidadosa, no sentido de não se caminhar ao encontro da ideia de que o elevado número de agressões é retrato de uma realidade contemporânea. Em verdade, um olhar pouco mais apurado evidencia que o que existe é um maior interesse dos especialistas e da sociedade neste tipo de fenômeno.

Lima e colaboradores (2008), por exemplo, atentam para esta questão ressaltando que a preocupação hoje presente em muitos dos discursos e práticas nem sempre existiu. De acordo com ele,

Essa recente percepção e consciência foi fruto de um trabalho incansável e articulado de diversos grupos, sendo os movimentos de mulheres e movimentos feministas os principais responsáveis pela remoção da pesada e empoeirada manta que mantinha em sigilo a dor e o medo de gerações de mulheres e famílias (Lima e colaboradores, 2008, p. 72).

Nesse contexto, e considerando o foco deste trabalho, é de fundamental importância que se destaque o papel assumido pela violência sexual neste conjunto das possíveis formas de violência. Minayo, Cavalcanti e Gomes (2006) apontam que esta é uma forma de violência silenciosa cujo encobrimento, quando comparado a outras formas de ação, é nitidamente maior, bem como o sofrimento causado por ela. Afirmam, ainda, que "as mulheres que passam por abuso físico e sexual [...] estão, de forma geral, sob maior risco de problemas de saúde do que as que passam [...] pela violência física" (Minayo e colaboradores, 2006, p. 33).

De acordo com Minayo e colaboradores (2006), no conjunto das violências cometidas contra a mulher, a violência sexual assume um significado especial, no sentido de que, não raramente, vem acompanhada de outras expressões. Para estas autoras, a violência sexual direcionada à mulher pode ser entendida como qualquer conduta onde o controle e a subordinação da sexualidade feminina são incorporados às regras que normatizam a prática sexual.

A violência sexual se apresenta a partir de diferentes modalidades de práticas sexuais - todas 
elas tendo em comum, no entanto, o fato de serem impostas à vítima. De acordo com Dell'aglio e Santos (2009), pode ser considerado abuso sexual qualquer toque genital ou comportamento sexual imposto à outra pessoa (com pelo menos cinco anos de idade a menos que o agressor) por meio de ameaça verbal e/ou física, e seriam exemplos desde as práticas eróticas, em que não se produz o contato sexual propriamente dito, como no voyeurismo, até diferentes tipos de contato sexual (com ou sem penetração).

Para Sant'Anna e Baima (2008, p. 347), no entanto, há uma falta de uniformidade nos dados estatísticos acerca da incidência de violência sexual e é praticamente impossível que se determine a real prevalência de abuso devido à subnotificação dos casos.

Para além de especificações, classificações ou divisões, o que se tem, em verdade, é que o abuso sexual não pode ser compreendido apenas como a consumação de um ato sexual ou como a imputação de qualquer outra forma de violência sexual. Este deve ser entendido como instrumento de dominação - um abuso de poder - no qual o que está em jogo vai além da satisfação do desejo sexual. Se este fosse o ponto central, o agressor poderia recorrer à prática do sexo consensual com um parceiro de sua faixa etária. 0 abuso sexual é, então, não apenas um ato de violência, mas acima de tudo um desejo de dominação (Schreiner, 2008).

Em cada cultura se lida com a violência sexual cometida contra mulher de uma determinada forma (Víctora, 1996; Winkler, 1994). Como aponta Víctora (1996, p.3), a cultura interfere diretamente na maneira como "os limites do corpo são percebidos e do entendimento sobre em quais circunstâncias uma mulher pensa que pode ou deve compartilhar o seu corpo com um homem" e, portanto, no entendimento da violência.

Entretanto, o que se tem observado, ao menos em nossa sociedade, é uma tendência à inibição do desejo feminino a partir da noção de que a sexualidade masculina encontra-se em uma esfera distinta em relação à sexualidade feminina: aquela envolveria uma necessidade que é da ordem do instinto, do biológico e, portanto, praticamente impossível de ser controlada (Payne, Lonsway e Fitzgerald, 1999). Tal concepção praticamente legitima a dominação e o controle cometidos pelos agressores contra suas vítimas.
0 que se pode observar também é que as mulheres têm sido colocadas na posição de culpadas pela violência sofrida e rotuladas não apenas como provocadoras, mas também como passivas - uma vez que comumente permanecem nesta condição de violência por muito tempo (Grossi,1994; Bessa, 1994). Assim, apesar da presença desta dita "marca cultural" (Porter, 1992; Womersley e Maw, 2009), ou seja, dessas diferenças com relação à compreensão do estupro cometido contra a mulher, o que se percebe é a frequente utilização do termo "consentimento" como forma de justificar o acontecido.

A reprodução destes papéis estereotipados de gênero por meio da família, da escola ou da mídia, acaba por se tornar, então, solo fértil para o acontecimento desta (e de tantas outras) formas de violência, pois acabam por validar a violência cometida contra a mulher. Um exemplo disso são os diversos casos de assassinato de mulheres por motivo de honra ao redor do mundo (Kulczycki \& Windle, 2011; Se'ver \& Yurdakul, 2001; Vandello, Cohen, Grandon, \& Franiuk, 2009).

Como apontam Carvalho e Arruda (2008), não há um isolamento entre os universos consensual e reificado, e é dentro deste contexto que esta pesquisa está inserida. Ressaltando a relevância de se compreender como as representações sociais podem ser construídas e apropriadas pelo senso comum a partir de trabalhos científicos.

Objetivando, então, investigar as representações sociais da violência sexual contra mulheres adultas, identificadas na produção científica nacional a respeito do tema, faz-se necessário apresentar resumidamente a Teoria das Representações Sociais.

\section{Estudos sobre Representação Social em Psicologia}

Foi com Serge Moscovici, em 1961, que a Teoria das Representações sociais teve seu início. Com $A$ Representação Social da Psicanálise (Moscovici, 1978), este autor inaugurou uma linha de pensamento a respeito da realidade que colocou o pensamento social, em toda sua dinâmica e diversidade, como fonte primordial de informação acerca de um fenômeno. De acordo com Arruda (2002, p.128), é nesta obra que está a "matriz da teoria" das representações.

De acordo com Alexandre (2004), Moscovici busca na Sociologia e na Antropologia, por meio

Psicol. Argum. 2014 abr./jun., 32(77), 9-18 
de Durkheim e Lévy-Bruhl, e também, na teoria da linguagem de Saussure e nas teorias de Piaget e Vygotsky os elementos que precisava para criação da teoria. Ainda de acordo com esse autor, "Moscovici resgata do emaranhado de conceitos sociológicos e psicológicos a definição de representação social, que para ele é 'uma modalidade de conhecimento particular que tem por função a elaboração de comportamentos e a comunicação entre os indivíduos'" (Alexandre, 2004, p.126).

De acordo com Silva e Souza (2010, p.34), o que a Teoria das Representações Sociais faz é investigar de que maneira os "grupos/indivíduos se apropriam do universo reificado e incorporam esse conhecimento ao cotidiano". Ou seja, a TRS se ocupa de compreender de que maneira torna-se familiar, para um grupo, o que outrora não era.

Ainda de acordo com Arruda (2002, p.129), a Teoria das Representações Sociais operacionaliza um "conceito para trabalhar com o pensamento social em sua dinâmica e em sua diversidade", pois parte da premissa de que "existem formas diferentes de conhecer e de se comunicar, guiadas por objetivos diferentes, formas que são móveis, e define duas delas, pregnantes nas nossas sociedades: a consensual e a científica" (p.130).

Para Moscovici (1978), a primeira diz respeito à vida cotidiana e suas conversações, são as ditas conversas "informais". A segunda, por sua vez, fala do universo científico, da elaboração de conhecimento a partir dos "especialistas".

A diferença, no caso, não significa hierarquia nem isolamento entre elas, apenas propósitos diversos. $\mathrm{O}$ universo consensual seria aquele que se constitui principalmente na conversação informal, na vida cotidiana, enquanto o universo reificado se cristaliza no espaço científico, com seus cânones de linguagem e sua hierarquia interna. Ambas, portanto, apesar de terem propósitos diferentes, são eficazes e indispensáveis para a vida humana (Arruda, 2002, p.130).

De acordo com Sá (2003), estão envolvidos nesse processo de familiarização dois processos sócio-cognitivos: objetivação e ancoragem, respectivamente. De acordo com Arruda (2002), por meio do processo de objetivação esclarece-se o processo de estruturação do conhecimento acerca de um objeto, da seguinte maneira: primeiro, seleciona-se e descontextualizam-se os elementos daquilo que se vai representar (enxugamento do excesso de informação a partir de nossa experiência e valores prévios); em seguida, "recosturam-se os fragmentos num esquema que se torna o núcleo figurativo da representação" (p.136). Desta maneira, o objeto que era desconhecido agora se torna natural e o ciclo da objetivação se completa e tem início uma segunda "fase": a ancoragem.

Mediante esse processo, se dá sentido àquele objeto que foi anteriormente naturalizado. Pela ancoragem, o objeto é reajustado de maneira a se encaixar numa determinada categoria preexistente e permite, pois, que se criem suas raízes sociais. De acordo com Oliveira e Werba (1998), ainda, esse processo de ancoragem não é destituído de juízo de valor

Abric (1994, 1998 ou 2001?) aponta que as Representações Sociais são visões de mundo que permitem aos grupos a compreensão da realidade a partir de seu próprio conjunto de referências, ou seja, a partir de construções que foram criadas e compartilhadas por aquele grupo. 0 conceito de campo representacional pode ser utilizado, aqui, para nos ajudar a compreender o movimento de construção das representações sociais - e, neste caso, dos autores dos trabalhos a serem analisados. Sobre isso, Camargo e Wachelke (2010) apontam que a expressão sistema ou campo representacional diz respeito à construção de uma unidade organizada a partir da relação de várias representações (que se relacionam mutuamente).

Para Monchietti, Lombardo, Sánchez e Krzemien (2010) é preciso lembrar, ainda, que o sistema representacional é heterogêneo e diverso, abrangendo visões contraditórias que coexistem num mesmo contexto representacional.

Tendo como base estes apontamentos, ressalta-se que este estudo se fundamenta na teoria proposta por Serge Moscovici (1978) e tem como objetivo principal compreender como se configuraram, nos últimos cinco anos, a produção científica sobre o abuso sexual cometido contra a mulher adulta no país.

Como objetivos específicos, esta pesquisa lista: 1) mapear a produção sobre a violência (abuso) sexual contra a mulher adulta, ressaltando-se os aspectos referentes à produção e divulgação do conhecimento sobre esse assunto; 2) apreender e sistematizar as representações sociais (o campo 
representacional) dessa temática no conjunto de produções científicas encontradas a partir da análise dos descritores que constam dos artigos; 3 ) verificar qual a contribuição da psicologia no que tange à construção destes conhecimentos.

\section{Método}

\section{Objeto de estudo}

A presente pesquisa teve como objetivo analisar a produção científica disponibilizada em artigos que abordassem a temática da violência sexual cometida contra a mulher adulta.

Para tanto, o objeto de estudo foi um conjunto de artigos disponibilizados pela biblioteca virtual Scientific Electronic Library Online - SciELO Brasil, e como critério para seleção utilizou-se artigos cujas palavras-chave continham os descritores: "abuso sexual", "violência sexual" e "violência contra a mulher".

Optou-se pela base de dados da SciELO Brasil na medida em que esta contém as informações referentes ao objeto de pesquisa, abrange diversificados periódicos científicos brasileiros e, portanto, atende ao objetivo proposto por esta pesquisa: o acesso às publicações que tratam da temática da violência sexual contra a mulher.

\section{Procedimento de coleta}

A seleção da produção científica resultou em 73 artigos de revistas diferentes (falando de abuso sexual de modo geral, incluindo crianças e adolescentes), datadas de 1994 a 2011.

Foram considerados como critérios de exclusão dos artigos a serem avaliados: a) a amostra (foram eliminados artigos que não trabalhavam com mulheres adultas), sendo selecionados 34 artigos; b) a data de publicação: optou-se por trabalhar com artigos publicados nos últimos cinco anos completos (2006 a 2011), sendo eliminados, nesse momento, 11 artigos; e c) a língua de publicação, sendo excluídos aqueles que não estavam em português ou em inglês. Ao final dessa seleção, restaram 22 artigos.

Devido à pequena quantidade de artigos encontrados e à proposta de análise, optou-se por acrescentar o descritor "estupro" na pesquisa. Foi realizada uma nova busca na base de dados e constaram 43 artigos disponíveis para leitura. Após a aplicação dos critérios de exclusão descritos anteriormente, restaram, desta amostra inicial, 13 artigos, que foram incluídos na seleção final.

\section{Organização e tratamento dos dados}

Os 35 artigos restantes foram numerados e se encontram referenciados pela letra A e pelo número correspondente, não expondo, pois, nem os títulos nem os autores dos trabalhos. Num primeiro momento, objetivou-se realizar um mapeamento geral dos artigos escolhidos para o trabalho. Para isso, realizou-se o levantamento de informações como: ano de publicação do trabalho, grande área de conhecimento do primeiro autor (a partir dos critérios do $\mathrm{CNPq}$ ), bem como sua vinculação institucional. Para isso, buscou-se a Plataforma Lattes do Conselho Nacional de Desenvolvimento Científico e Tecnológico - CNPq ou as próprias informações presentes nos artigos, quando os autores não possuíam cadastro na Plataforma Lattes, de forma semelhante ao realizado por Silva e Souza (2010).

Finalizada esta etapa, iniciou-se a análise dos objetivos gerais dos artigos selecionados por meio da Análise de Conteúdo. Esta é uma técnica cujo objetivo consiste em interpretar o conteúdo manifesto das comunicações (Berelson, 1971, citado por Lima, 2008). Isso acontece mediante uma descrição objetiva, sistemática e quantitativa.

Esta etapa requer que todos os dados sejam transcritos em sua totalidade e revisados, para que, então, o corpus final seja analisado (Bardin, 1977). Nascimento e Menandro (2005) sugerem, ainda, as seguintes etapas:

Uma primeira leitura do corpus com anotação sobre possíveis elementos recorrentes;

Uma segunda leitura do corpus, objetivando uma pré-categorização;

Terceira leitura, objetivando a construção de categorias mais gerais com palavras e frases relacionadas ao tema;

Agrupamento dos elementos em subcategorias;

Por fim, a contagem da frequência final dos elementos tanto nas subcategorias quanto nas categorias gerais. 


\section{Resultados e Discussão}

Para facilitar a compreensão dos resultados obtidos com a pesquisa, estes serão apresentados em tabelas, seguidas de uma discussão sobre a temática proposta. A Tabela 1 apresenta a distribuição dos artigos pelo ano de sua publicação. Observa-se, que dentro do período de tempo escolhido para análise, os últimos cinco anos, há um crescimento da publicação sobre violência sexual cometida contra a mulher adulta a partir do ano de 2007, em que 8 artigos sobre o tema são publicados.

Tabela 1 - Distribuição dos artigos por ano de publicação

\begin{tabular}{ccc}
\hline ANO DE PUBLICAÇÃO & ARTIGOS (N) & PORCENTAGEM \\
\hline 2006 & 05 & $14,28 \%$ \\
2007 & 08 & $22,85 \%$ \\
2008 & 10 & $28,57 \%$ \\
2009 & 05 & $14,28 \%$ \\
2010 & 04 & $11,43 \%$ \\
2011 & 03 & $8,57 \%$ \\
\hline
\end{tabular}

0 ápice de publicações encontra-se no ano de 2008, com 10 artigos publicados. Estes dois anos, na verdade, concentram juntos mais de $50 \%$ das publicações do período de tempo estimado $(51,42 \%$ mais exatamente).

A Tabela 2 apresenta a distribuição dos autores a partir da Grande Área de Conhecimento adotada pelo CNPq. Este tem em sua classificação das áreas do conhecimento um total de 9 categorias, sendo elas: as Ciências Exatas e da Terra, as Ciências da Saúde, as Ciências Biológicas, as Engenharias, as Ciências Agrárias, as Ciências Sociais Aplicadas, a Linguística, Letras e Artes, as Ciências Humanas, e Outros.

Tabela 2 - Grande Área de Conhecimento organizada pelo Currículo Lattes do primeiro autor

\begin{tabular}{lc}
\hline Grande área de conhecimento & Autores (N) \\
\hline Ciências da Saúde & 23 \\
Ciências Humanas & 07 \\
Ciências Biológicas & 01 \\
Não Identificado & 04 \\
\hline
\end{tabular}

Ressalta-se que, apesar de no processo de criação de banco de dados não se ter restringido a busca, por meio das palavras-chave, a determinadas áreas do conhecimento, só foram encontrados artigos nas seguintes áreas: Ciências Humanas, Ciências da Saúde e Ciências Biológicas. Outro ponto a ser considerado refere-se ao fato de que alguns artigos foram escritos por autores que não disponibilizaram seus dados, de modo a não ser possível a sua classificação em uma das áreas já anteriormente citadas.

$\mathrm{A}$ área que concentrou o maior número de autores com artigos publicados foi Ciências da Saúde, com 23 autores (65,71\%), seguido pela área de Ciências Humanas, com 7 autores (20\%). Ressaltase, ainda, que dentro da Grande Área Ciências da Saúde há um predomínio da área de medicina e enfermagem.

\section{Tabela 3 - Universidade de filiação do primeiro autor}

\begin{tabular}{lc}
\hline UNIVERSIDADE DE FILIAÇÃO DO & AUTORES (N) \\
PRIMEIRO AUTOR & \\
\hline $\begin{array}{l}\text { Universidade Federal de São Paulo - } \\
\text { UNIFESP }\end{array}$ & 06 \\
$\begin{array}{l}\text { Universidade Estadual de Campinas - } \\
\text { UNICAMP }\end{array}$ & 04 \\
$\begin{array}{l}\text { Universidade Federal do Rio Grande do } \\
\text { Sul - UFRGS }\end{array}$ & 03 \\
$\begin{array}{l}\text { Universidade Federal do Piauí - UFPI } \\
\text { Universidade de São Paulo - USP }\end{array}$ & 02 \\
$\begin{array}{l}\text { Universidade Federal } \\
\text { de Santa Catarina - UFSC }\end{array}$ & 05 \\
Outros & 02 \\
\hline
\end{tabular}

A Tabela 3 mostra a distribuição dos artigos com relação à instituição à qual o primeiro autor está vinculado. Observa-se que há uma distribuição desigual em relação às regiões, havendo concentração majoritária dos autores na região Sudeste, ainda predominante na produção e publicação sobre a violência sexual. São Paulo é o estado que mais se destaca nesse cenário, ocupando os três primeiros postos no que tange à vinculação dos autores a universidades de São Paulo ou da região. Como observado por Silva e Souza (2010) em seu trabalho sobre a publicação científica acerca da pobreza, também aqui se pode inferir que a concentração dos grandes institutos de pesquisa e programas de pós-graduação nessa região do país se relaciona 
diretamente ao fato de que esta continue sendo a região que mais se destaca.

Ressalta-se que a categoria "Outros" contém apenas as Universidades que apresentaram somente um autor vinculado e, por isso, não foram consideradas como relevantes para análise de dados neste momento.

A concentração da produção sobre esta temática no estado de São Paulo faz surgir indagações como: o maior interesse desse estado pelo tema está associado a uma maior incidência desse tipo de violência? Estaria faltando incentivo ou acesso à publicação em outros estados brasileiros? A baixa produção também pode ser um indicativo de pouca preocupação com o tema ou de que tais casos de violência contra mulheres adultas não são considerados problemas sociais relevantes.

Dada esta primeira parte de apresentação dos dados e de suas características principais, parte-se agora para apresentação das categorias encontradas nos dados. Como dito anteriormente, para que esta análise fosse realizada, utilizaram-se os objetivos gerais de cada artigo encontrado. Este foi decomposto em suas palavras principais, tal como as palavras-chave de um artigo, e depois analisados mediante uma análise temática de conteúdo (ver Tabela 4).

Pensando-se a partir de categorias, os principais temas identificados como recorrentes no estudo científico da violência sexual:

1. Os personagens envolvidos na violência: esta categoria engloba todos os termos utilizados nos artigos para descrever aqueles que de alguma forma estão envolvidos na violência, seja como agressor ou como vítima.

2 . 0 contexto em que a violência ocorreu: nesta categoria estão todas as características relativas ao contexto em que houve a violência, seja no presente ou no passado, seja num plano individual ou coletivo.

3. As práticas relacionadas à violência: nesta categoria estão inclusos todos os termos referentes às práticas ou atitudes que podem ser referenciadas a este tipo de violência por parte dos profissionais que de alguma forma tenham contato ou com as vítimas, ou agressores, a família ou mesmo com outros profissionais.

É importante destacar que, nos últimos anos, observou-se o início de um progressivo crescimento

Tabela 4 - Categorização das evocações

\begin{tabular}{|c|c|c|c|}
\hline CATEGORIA & CONTEÚDO & $\mathbf{F}$ & $\%$ \\
\hline $\begin{array}{l}\text { 1. Envolvidos na } \\
\text { Violência }\end{array}$ & $\begin{array}{l}\text { Definição: sujeitos que, de alguma forma, fazem ou fizeram parte da violência. } \\
\text { Ex.: Mulher; vítimas; agressores; parceiro-íntimo; acusados; mulher-vítima. }\end{array}$ & 31 & $15,19 \%$ \\
\hline 2. Contexto & $\begin{array}{l}\text { Definição: elementos que fazem parte do contexto em que a violência ocorreu. } \\
\text { Ex.: Contextos-socioculturais; consumo-de-álcool-e-drogas; fatores-associados; históri- } \\
\text { co; personalidade. }\end{array}$ & 27 & $13,23 \%$ \\
\hline 3. Práticas & $\begin{array}{l}\text { Definição: ações realizadas por quem trabalha ou lida com a violência, em todos os seus } \\
\text { níveis. } \\
\text { Ex.: Intervenção; acolhimento; cuidar; cuidados; registros; oficinas-de-auto-estima; } \\
\text { procedimentos; Protocolo-de-Enfermagem; atendimento. }\end{array}$ & 26 & $12,74 \%$ \\
\hline 4. Tipos de Violência & $\begin{array}{l}\text { Definição: tipos de violência encontrados nos trabalhos pesquisados. } \\
\text { Ex.: Agressões; genocídio; crime; abuso-sexul; estupro; violência; violência-sexual. }\end{array}$ & 23 & $11,27 \%$ \\
\hline 5. Consequências & $\begin{array}{l}\text { Definição: consequências da violência, seja para o agressor ou para a vítima. } \\
\text { Ex.: Agravos; sorologias; Aids; resultados; HIV; aborto. }\end{array}$ & 09 & $4,41 \%$ \\
\hline 6. Profissionais & $\begin{array}{l}\text { Definição: Profissionais que de alguma forma lidam com a violência relatada. } \\
\text { Ex.: Enfermagem; profissionais; enfermeiros. }\end{array}$ & 09 & $4,41 \%$ \\
\hline 7. Instituições & $\begin{array}{l}\text { Definição: espaços que lidam com a violência ou com suas vítimas. } \\
\text { Ex.: Hospital-de-emergência; Delegacia-de-Defesa-da-Mulher; Ministério-Público; } \\
\text { Varas-Criminais. }\end{array}$ & 09 & $4,41 \%$ \\
\hline 8. Educação/Ensino & $\begin{array}{l}\text { Definição: de que maneira o tema tem sido retratado no meio acadêmico. } \\
\text { Ex.: Pesquisa-etnográfica; conhecimento; politização; implicações-teóricas. }\end{array}$ & 07 & $3,43 \%$ \\
\hline 9. Sentimentos & $\begin{array}{l}\text { Definição: sentimentos e percepção das pessoas envolvidas direta ou indiretamente na } \\
\text { violência. } \\
\text { Ex.: Vivência-no-cuidar; sentimentos-contratransferenciais; percepção-de-saúde. }\end{array}$ & 05 & $2,45 \%$ \\
\hline
\end{tabular}

Psicol. Argum. 2014 abr./jun., 32(77), 9-18 
do movimento contra o abuso sexual, encarando-o como um problema de caráter não apenas privado, mas fundamentalmente social. Paulatinamente, o que se percebeu foi o direcionamento da atenção de pesquisadores, autoridades e da sociedade como um todo para este tema, antes velado (Araújo, 2002). Entretanto, para Pimentel e Pandjiarjian (1998), assim como para Souza, Assis e Alzuguir (2002), ainda há uma defasagem teórico-metodológica quanto à discussão desta temática; o que de alguma forma foi confirmado neste trabalho.

No entanto, o que mais chamou atenção foi a ausência de trabalhos produzidos na área da Psicologia nestes últimos cinco anos de produção científica sobre a violência sexual cometida contra a mulher. 0 que se pode observar é que a participação maior de autores da área se localiza em trabalhos referentes à violência sexual cometida contra a criança e o adolescente, mas não contra esta parcela específica da população.

\section{Conclusão}

O presente estudo buscou identificar o campo representacional acerca da violência sexual cometida contra a mulher adulta na produção científica nacional. Ele representa um esforço no sentido de estimar como o meio acadêmico percebe o tema. Acreditamos que o objetivo do estudo tenha sido alcançado. No entanto, é necessário destacar uma possível limitação. Foram consideradas apenas as publicações indexadas no banco de dados Scielo. Tal decisão pode ter excluído artigos científicos sobre o tema publicados por pesquisadores brasileiros em revistas estrangeiras. Acredita-se, entretanto, que tal limitação não invalide os resultados encontrados, cuja principal contribuição foi discutir a percepção dos pesquisadores nacionais sobre a violência sexual.

Ao se contextualizar a produção científica, identificando qual a representação social da violência sexual nos artigos encontrados, pode-se deparar não apenas com os dados que aqueles que se dedicam à área imaginam encontrar (tais como a discussão sobre a vítima da violência), mas também com outras questões que se poderia dizer que foram inesperadas.

Dois pontos principais precisam ser destacados nesse sentido: o primeiro diz respeito ao próprio conteúdo das categorias, que apresentam de maneira clara o modo como tem sido representada a questão da violência sexual contra a mulher adulta em artigos científicos no país. Tal conteúdo indica uma forte preocupação da comunidade científica com os atendimentos e tratamentos médicos especializados para as vítimas de violência. No entanto, algumas lacunas são claramente percebidas, sendo necessário um maior interesse e aprofundamento dos pesquisadores no que diz respeito aos aspectos psicológicos desse tipo de violência.

O segundo ponto a ser destacado se refere à já dita escassa presença da Psicologia nas produções sobre o tema no meio científico. Tal como encontrado por Silva e Souza (2010, p.48) em seu trabalho sobre a produção científica acerca da pobreza, "os resultados sobre a incipiente produção da Psicologia [...] sugerem a necessidade de se pensar o papel da produção da área sobre as questões sociais", bem como a necessidade de uma maior articulação com outras áreas de conhecimento.

Pesquisas futuras poderiam investigar a percepção da autoimagem corporal e autoestima das mulheres vítimas de violência; os aspectos psicossociais envolvidos na prevenção, além do funcionamento de grupos de apoio às vítimas. Neste sentido, é importante enfatizar a necessidade de mais pesquisas serem conduzidas sobre o tema no Brasil.

\section{Referências}

Abric, J. C. (1994). Pratiques sociales et représentations. Paris: Presses Universitaires de France.

Abric, J.C. (1998). A abordagem estrutural das Representações Sociais. In A. S. Moreira, \& D. C. Oliveira. (Orgs.). Estudos interdisciplinares de Representação Social (pp. 27-38). Goiânia: Editora AB.

Abric, J.C. (2001). Les représentations sociales: aspects théoriques. In Abric, J. C. (Org.). Pratique sociales et représentations (pp. 11-36). Paris: PUF.

Alexandre, M. (2004). Representação Social: uma genealogia do conceito. Comum, 23, 122-138.

Araújo, M. F. (2002). Violência e abuso sexual na família. Psicologia em Estudo, 7 (2), 3-11.

Arruda, A. (2002). Teoria das representações sociais e teorias de gênero. Cadernos de Pesquisa, 117, 127-147. 
Bardin, L. (1977). Análise de Conteúdo. Lisboa: Edições 70.

Bessa, K. A. (1994). 0 crime de sedução e as relações de gênero. Cadernos Pagu, 2, 175-196.

Camargo, B. V., \& Wachelke, J. (2010). The study of social representation systems: relationships involving representations on aging, AIDS and the body. Papers on Social Representations, 19 (2), 1-21.

Carvalho, J. G S., \& Arruda, A. (2008). Teoria das representações sociais e história: um diálogo necessário. Paidéia (Ribeirão Preto), 18 (41), 445-465.

Chauí, M. (1985). Participando do debate sobre mulher e violência. In Perspectivas Antropológicas da Mulher (vol. 4, pp. 25-62). Rio de Janeiro: Zahar.

Dell'aglio, D. D., \& Santos, S. S. (2009). Revelação do abuso sexual infantil: reações maternas. Psicologia: Teoria e Pesquisa, 25 (1), 85-92.

Giffin, K. (1994). Violência de gênero, sexualidade e saúde. Caderno de Saúde Pública, 10, 146-155.

Grossi, M. (1994). Novas/velhas violências contra a mulher no Brasil. Revista Estudos Feministas, 1, 462 - 472.

Kulczycki, A., \& Windle, S. (2011). Honor killings in the Middle East and North Africa: A systematic review of the literature. Violence Against Women, 17 (11), 1442-1464.

Lima, L. (2008). Programa Alceste, primeira lição: a perspectiva pragmatista e o método estatístico. Revista de Educação Pública, 33, 83-97.

Lima, D. C., Buchele, F., \& Climaco, D. A. (2008). Homens, gênero e violência contra a mulher. Saúde \& Sociedade, 17(02), 69-81.

Minayo, M. C. S; Cavalcanti, L. F., \& Gomes R. (2006). Representações sociais de profissionais de saúde sobre violência sexual contra a mulher: estudo em três maternidades públicas municipais do Rio de Janeiro, Brasil. Caderno Saúde Pública, 22(1), 31-39.

Monchietti, A., Lombardo, E., Sánchez, M. L., \& Krzemien, D. (2010). Funcionamiento cognitivo en la vejez: Su campo representacional. Límite, 5 (22), 89-110.

Moscovici, S. (1978). A representação social da psicanálise. Rio de Janeiro: Zahar.

Narvaz, M., \& Koller, S. H. (2006). Mulheres vítimas de violência doméstica: compreendendo subjetividades assujeitadas. PSICO, 37(1), 7-13.
Nascimento, A., \& Menandro, P. (2005). Memória social e saudade: especificidades e possibilidades de articulação na análise psicossocial de recordações. Memorandum: memória e história em Psicologia, 8, 5-19.

Oliveira, F., \& Werba, G. (1998). Representações Sociais. In Strey, M., \& Jacques, M. da G. Psicologia Social Contemporânea. Porto Alegre: Vozes.

Organização Mundial de Saúde - OMS. (2005), Alianza para la prevención de la violência: Promoción de un compromiso mundial en pro de la prevención de la violencia. Recuperado de http://www.who.int/ violenceprevention/publications/vpabroch_es.pdf

Payne D., Lonsway K., \& Fitzgerald L. (1999). Rape myth acceptance: Exploration of its structure and its measurement using the Illinois rape myth acceptance scale. Journal of Research in Personality, 33, 27-68.

Pimentel, S., Schritzmeyer, A. L., \& Pandjiarjian, V. (1998). Estupro: crime ou "cortesia"? Abordagem sócio-jurídica de gênero. Porto Alegre: Sergio Antonio Fabris.

Porter, R. (1992). Estupro - Será que ele tem um significado histórico?. In S. Tomaselli, \& R. Porter. (Orgs.) Estupro (pp. 207-227). Rio de Janeiro: Rio Fundo.

Sá, C. P. (2003). O núcleo central das Representações Sociais. Petrópolis: Vozes.

Sant'Anna, A., \& Baima, A. S. (2008). Indicadores clínicos em psicoterapia com mulheres vítimas de abuso sexual. Psicologia: Ciência e Profissão, 28 (4), 728-74.

Schreiner, M. T. (2008). 0 abuso sexual numa perspectiva de gênero: o processo de responsabilização da vítima. Fazendo Gênero 8 - Corpo, Violência e Poder, Florianópolis. Recuperado de http://www.fazendogenero.ufsc.br/8/sts/ST41/Marilei_Teresinha_ Schreiner_41.pdf.

Sev'er, A., \& Yurdakul, G. (2001). Culture of honor, culture of change: A feminist analysis of honor killings in rural Turkey. Violence Against Women, 7, 964-998.

Silva, R. D. M., \& Souza, L. (2010). A produção acadêmica brasileira acerca da pobreza na perspectiva das Representações Sociais. Mental (Barbacena), 7, 29-48.

Souza, E. R.; Assis, S. G. \& Alzuguir, F. C. V. (2002). Estratégias de atendimento aos casos de abuso sexual infantil: um estudo bibliográfico. Revista Brasileira de Saúde Materno-Infantil, 2 (2), 105-116. 
Scarpati, A. S., Rosa, E. M., \& Guerra, V. M.

Vandello, J. A., Cohen, D., Grandon, R., \& Franiuk, R. (2009). Standy by your man: Indirect prescriptions for honorable violence and feminine loyalty in Canada, Chile, and the United States. Journal of Cross-Cultural Psychology, 40 (1), 81-104.

Víctora, C. G. (1996). Os limites do corpo sexual: um estudo sobre experiências corporais de mulheres inglesas. Corpus - Cadernos do NUPACS, 2, 1-20.
Winkler, C. (1994). Rape trauma: Contexts of meaning. In T. J. Csordas (Ed.). Embodiment and experience. Cambridge: Cambridge University Press.

Womersley, G., \& Maw, A. (2009). Contextualising the experiences of South African women in the immediate aftermath of rape. Psychology in Society, 38, 40-60. 